\title{
Low-energy hadronic cross sections measurements at BABAR and implication for the $g-2$ of the muon
}

\author{
Denis Bernard*广 \\ LLR, CNRS/IN2P3 \\ E-mail: denis.bernardellr.in2p3.fr
}

The BABAR Collaboration has an intensive program studying the cross sections of hadron production in low-energy $e^{+} e^{-}$annihilation, accessible via initial-state radiation. Our measurements allow a significant improvement in the precision of the predicted value of the muon anomalous magnetic moment. These improvements are necessary for shedding light on the current $>3$ sigma difference between the predicted and the experimental values. We have published results on a number of processes with two to six hadrons in the final state, and other final states are currently under investigation. We report here on the most recent results obtained by analysing the entire $B A B A R$ dataset.

XXIV International Workshop on Deep-Inelastic Scattering and Related Subjects

11-15 April, 2016

DESY Hamburg, Germany

\footnotetext{
* Speaker.

${ }^{\dagger}$ The BaBar Collaboration.
} 
Even though the standard model of particle physics has been tested in depth by several generations of researchers and found to be extremely standard indeed, some hints of new physics are cropping out here and there, most notably the long-pending 3-ish standard deviation discrepancy between the predictions and the measurement of the muon anomalous magnetic moment $a_{\mu}$. The experimental information originates from one single precise measurement, while the prediction uncertainty is dominated by two main contributions, the hadronic vacuum polarization (VP) modification of the photon propagator and the hadronic light-by-light scattering (LbL). The leading-order (LO) VP contribution to $a_{\mu}$ is obtained using the dispersion relation and the optical theorem as the integral as a function of energy of an expression that involves the ratio of the $e^{+} e^{-} \rightarrow$ hadrons cross section to the pointlike muon pair cross section. The former is extracted from experimental data for individual hadronic final states at low energies, and from perturbative QCD at high energies.

The BABAR experiment at SLAC has a programme of systematic measurement of the production of the lowest-rest-mass hadronic final states, those that contribute most significantly to the integral. To that purpose, we use a method in which, while the PEP-II storage ring is operated at a constant energy in the center of mass system, $\sqrt{s}$, of about $10.6 \mathrm{GeV}$, events are reconstructed and selected which have been produced with a hadronic final state together with a high-energy photon which may (photon tagging) or may not (no tagging) be observed. In our kinematic configuration the photon is almost always emitted by the electron or by the positron of the initial state, hence the name "initial-state radiation" (ISR). The cross section for the direct $e^{+} e^{-} \rightarrow f$ production of a final state $f$ at an energy $\sqrt{s^{\prime}}$ is then extracted from the differential cross section of the ISR production of the state $f$ with invariant mass $\sqrt{s^{\prime}}$. The programme is almost completed and has lead to a number of first measurements and to an improvement of up to a factor of three of the uncertainties on the contributions of individual channels to $a_{\mu}$. The uncertainty on $a_{\mu}^{\mathrm{VP}}$ is now of similar magnitude as that on $a_{\mu}^{\mathrm{LbL}}$.

On the experimental side, two projects aiming at a measurement of $a_{\mu}$ with an improved precision are in preparation at Fermilab and at J-PARC: their results are eagerly awaited !

The full text is available at D. Bernard, the BABAR Collaboration, [arXiv:1604.07641 [hep-ex]]. 